\title{
Nucleosynthesis constraints on the faint vector portal
}

\author{
Anthony Fradette ${ }^{1}$, Maxim Pospelov ${ }^{1,2}$, Josef Pradler ${ }^{3, a}$, and Adam Ritz ${ }^{1}$ \\ ${ }^{1}$ Department of Physics and Astronomy, University of Victoria, Victoria, BC V8P 5C2, Canada \\ ${ }^{2}$ Perimeter Institute for Theoretical Physics, Waterloo, ON N2J 2W9, Canada \\ ${ }^{3}$ Institute of High Energy Physics, Austrian Academy of Sciences, A-1050 Vienna, Austria
}

\begin{abstract}
New Abelian $U(1)^{\prime}$ gauge bosons $V_{\mu}$ can couple to the Standard Model through mixing of the associated field strength tensor $V_{\mu \nu}$ with the one from hypercharge, $F_{\mu \nu}^{Y}$. Here we consider early Universe sensitivity to this vector portal and show that the effective mixing parameter with the photon, $\kappa$, is being probed for vector masses in the $\mathrm{GeV}$ ballpark down to values $10^{-10} \lesssim \kappa \lesssim 10^{-14}$ where no terrestrial probes exist. The ensuing constraints are based on a detailed calculation of the vector relic abundance and an in-depth analysis of relevant nucleosynthesis processes.
\end{abstract}

\section{Introduction}

The origins of our Universe may well be rooted in inflation or alternative cataclysmic scenarios that regard the very earliest moments of existence. However, despite the impressive success of observational cosmology over the past decades, the earliest true direct window into the beginnings remain observations of light element abundances. They concern the epoch of primoridal nuclear transformations at cosmic times $t \gtrsim 1 \mathrm{~s}$. The overall concordance of the Big Bang nucleosynthesis (BBN) predictions with the observationally inferred primordial values is one of the most impressive successes of modern day cosmology and particle physics. Today, BBN is used as a toolbox to put models of new physics to a stringent test [1], whenever they predict some interference with the the standard processes in the observable sector at $t \gtrsim 1 \mathrm{~s}$.

Under the assumption of a canonical sequence of cosmological events, the Universe emerged from inflation and baryogenesis much prior to BBN. Such sequence then allows one to put stringent constraints on very weakly interacting sectors of new physics beyond the Standard Model (SM). The kinetic mixing of a new $U(1)^{\prime}$ vector $V_{\mu}$ with hypercharge $F_{\mu \nu}^{Y} V^{\mu \nu}$ is of particular interest as the mixing with the photon leads to numerous experimental consequences and much attention was devoted to this vector portal in recent years [2]. Below the electroweak scale, the coupling of $V$ to the SM is essentially given by its mixing with the photon [3],

$$
\mathcal{L}_{\mathrm{V}}=-\frac{\kappa}{2} F_{\mu \nu} V^{\mu \nu}=e_{\kappa} V_{\mu} J_{\mathrm{em}}^{\mu}
$$

With $\kappa$ and $m_{V}$ being the only free parameters, the model provides a simple, and technically natural prototype scenario for a light, weakly interacting new particle sector. In

\footnotetext{
a e-mail: josef.pradler@oeaw.ac.at
}

the following we will concentrate on a Stückelberg origin of $m_{V}$ that allows to maintain gauge invariance in $U(1)^{\prime}$ without complicating the phenomenology by hidden Higgs particles $h^{\prime}$; see e.g. [4] for the phenomenology and [5] for cosmological constraints on the latter scenario.

The SM decay modes of $V$ are well known. When hadronic decays are kinematically accessible, one can use experimental data on the $R$-ratio to infer couplings to photons in the time-like direction, and hence to determine the decay rate $\Gamma_{V}$ and all branching ratios. Below the di-muon threshold and for $m_{V}>1 \mathrm{MeV}$ the vector $V$ decays to electron-positron pairs only, thereby setting its principal lifetime,

$$
\tau_{V} \simeq \frac{3}{\kappa^{2} \alpha m_{V}}=270 \mathrm{~s} \times \frac{1 \mathrm{GeV}}{m_{V}}\left(\frac{10^{-12}}{\kappa}\right)^{2},
$$

where $\alpha$ is electromagnetic fine structure constant. In the following the cosmological consequences of $U(1)^{\prime}$ vectors with masses in the $\mathrm{MeV}-\mathrm{GeV}$ range, and lifetimes long enough for the decay products to directly influence primordial nucleosynthesis are explored. These vectors have a parametrically small coupling to the electromagnetic current, and thus an extremely small production cross section for $e^{+} e^{-} \rightarrow V \gamma, \sigma_{\text {prod }} \sim \kappa^{2} \pi \alpha^{2} / s \sim 10^{-54} \mathrm{~cm}^{2}$ where we took $\sqrt{s}=200 \mathrm{MeV}$ and $\kappa=10^{-12}$ from above. Such small couplings render these vector states completely undetectable in terrestrial particle physics experiments.

Despite the tiny production cross section, any charged SM state that is populated in appreciable number in the early Universe at temperature $T \sim m_{V}$ may yet emit $V$. With the above ballpark numbers in (2), parametric estimates suggest that an amount of $\mathrm{MeV}$ /baryon may be stored in $V$-particles. Followed by late decays back to SM states, visible energy is therefore being injected into the primordial plasma at levels that are probed by $\mathrm{BBN}$. The 


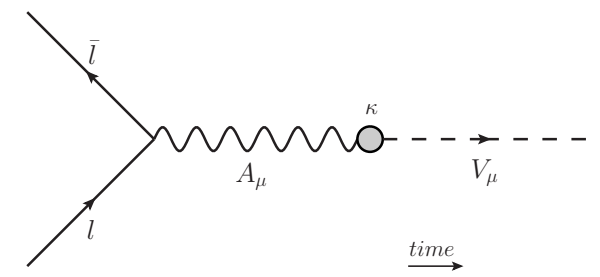

Figure 1. Dark photon inverse decays are the leading contribution to sub-Hubble production rates in the calculation of the $V$ relic density.

early Universe is therefore likely to be the only "laboratory" where such very dark photons with $\kappa \sim 10^{-12}$ and smaller are being probed. Here we report on the detailed analysis performed in [7] where also CMB limits on even later decays were considered. Previous partial discussions of cosmological signatures of decaying dark photons may also be found in $[5,6]$.

In the following we assume no other light states $\chi$ that are charged under $U(1)^{\prime}$. Therefore, there are no decays $V \rightarrow \chi \bar{\chi}$ that potentially drain visible modes and thereby ameliorate the derived limits from BBN. Using some recent insight about the in-medium production of dark vectors [8,9] (see also [10]) we first discuss the production of dark vectors in the next section and explore constraints on $V$-decays into SM in Sec. 3 before concluding with Sec. 4.

\section{Abundance prior to decay}

The relic abundance of weakly coupled dark photons prior to their decay is obtained through a calculation of the leakage from the observable sector to the hidden sector with sub-Hubble rates, $\Gamma_{\text {prod }} / H \ll 1$. This "freeze-in" process is dominated by inverse decays of $V$, through the coalescence of $e^{ \pm}, \mu^{ \pm} \ldots$, and, similarly, through hadronic contributions; see the illustration in Fig. 1.

The Boltzmann equation for the total number density of $V$ takes the form

$$
\dot{n}_{V}+3 H n_{V}=C \text {. }
$$

The right hand side is the collision integral and provided that $V$ never reaches thermal equilibrium, it gives the number of $V$ states emitted per unit volume and unit time. In the Maxwell-Boltzmann approximation for the SM distribution functions, and in the limit that only electrons coalesce, the integration can be done analytically $[5,7]$ (see also [6])

$$
C \simeq \frac{3}{2 \pi^{2}} \Gamma_{V \rightarrow e \bar{e}} m_{V}^{2} T K_{1}\left(m_{V} / T\right)
$$

Here, $\Gamma_{V \rightarrow e \bar{e}}=\kappa^{2} \alpha m_{V} / 3$ is the decay width of $V$ to electrons, up to corrections $\left(m_{e} / m_{V}\right)^{2}$ and $K_{1}$ is the modified Bessel function of the second kind. In terms of the number of $V$ particles normalized to entropy density, $Y_{V}=n_{V} / s$, the cosmic time integral in (3) for the final "freeze-in" abundance can be performed explicitly,

$$
Y_{V}^{(e)}=\frac{9}{4 \pi} \frac{m_{V}^{3} \Gamma_{V \rightarrow e \bar{e}}}{(H s)_{T=m_{V}}} .
$$

While further leptonic production channels are easy to be included into (5), hadronic production channels require assumptions about the primordial hadron gas and the strength of interaction with photons, denoted by $\alpha_{\text {eff }}$. At temperatures above the QCD confinement scale $T_{c} \sim$ $200 \mathrm{MeV}$ light quarks are deconfined and individual quark contributions can be added to $Y_{V}$ in a straightforward manner. Below $T_{c}$ one may use a free gas of mesons as an approximation to the hadronic (non-baryonic) particle content in the early Universe. The production via inverse charged pion and kaon decays $\left\{\pi^{+} \pi^{-}, K^{+} K^{-}\right\} \rightarrow V$ can then be included using a scalar QED model with effective coupling strengths like $\alpha_{\text {eff }}^{\pi \pi}\left(m_{V}\right)=\kappa^{2} \alpha^{\pi \pi}\left(\sqrt{s}=m_{V}\right)$ where $\alpha^{\pi \pi}$ is extracted from $\mathrm{BaBar}$ cross section measurements of $e^{+} e^{-} \rightarrow \gamma^{*} \rightarrow \pi^{+} \pi^{-}(\gamma)$ [11], and similarly for charged kaons [12].

Finally, there is a possibility of resonant production of $V$ by virtue of the thermal bath. Such in-medium effects may be cast into an effective mixing angle,

$$
\kappa_{T, L}=\frac{\kappa}{\left|1-\Pi_{T, L} / m_{V}^{2}\right|},
$$

with $\Pi_{T, L}$ being the transverse $(T)$ and longitudinal $(L)$ photon polarization functions in the primordial, isotropic plasma. The expressions for $\Pi_{T, L}$ can e.g. be found in [13]; the longitudinal polarization function [8] used here is, $\Pi_{L}^{\text {here }}=m_{V}^{2} /\left(\omega^{2}-m_{V}^{2}\right) \Pi_{L}^{\text {Ref. [13] }}$ and $\omega$ is the (dark) photon energy. Equation (6) informs us about the condition of resonant dark photon production,

$$
\operatorname{Re} \Pi_{T, L}\left(\omega, T_{r, T, L}\right)=m_{V}^{2} .
$$

The condition depends on temperature $T$ as $\Pi_{T, L}$ are proportional to the plasma frequency, $\omega_{P}(T)$. Most importantly, the resonance temperature $T_{r, T, L}(\omega)$ as a function of frequency $\omega$ is parametrically larger than $m_{V}$ with a minimum frequency at which the resonance can happen,

$$
T_{\mathrm{r}, \min }=m_{V}\left[\frac{3}{2 \pi \alpha}\right]^{1 / 2} \simeq 8 m_{V} .
$$

Thus resonances occur at parametrically larger temperatures (by $\alpha^{-1 / 2}$ ) than $m_{V}$, for which $H(T)$ is significantly larger than at $T \simeq m_{V}$ at which the $V$ freeze-in production has its biggest contribution. Therefore, resonant contributions to $Y_{V}$ do not alter the picture drastically though numerically they may constitute as much as $30 \%$.

After production, the momentum of $V$ redhifts quickly so that at the time of decay the energy of $V$ is to good approximation given by the rest mass, $E_{V}=m_{V}$. The decay deposits this energy into leptons, hadrons, and hadronic resonances. The energy prior to decay that is stored per baryon is therefore given by

$$
E_{\text {p.b. }}=m_{V} Y_{V} \frac{s_{0}}{n_{b, 0}}
$$


where $n_{b, 0} / s_{0}=0.9 \times 10^{-10}$ is the baryon-to-entropy ratio today. Equipped with $E_{\text {p.b. }}$ as a function of $m_{V}$ and $\kappa$ following the detailed calculations of the $V$ "freeze in" abundance in [7], we may now explore its consequences for BBN.

\section{$3 V$-decays during BBN}

Primordial nucleosynthesis predictions are affected for dark photon decays with cosmic lifetime $t \gtrsim 1 \mathrm{~s}$ or larger. Ensuing constraints are then governed by a combination of lifetime and abundance, both being complementary with respect to the vector mass: $\tau_{V}\left(Y_{V}\right)$ decreases (increases) with larger $m_{V}$. From this one expects constraints as localized islands in those parameters where the epoch of primordial nucleosynthesis exhibits its greatest sensitivity.

\subsection{Major effects and treatment}

The effects on BBN are understood by considering electromagnetic and hadronic energy injection separately. Prior to decay, the $V$ abundance relative to baryons is substantial, $n_{V} / n_{b} \lesssim 10^{8}$ for $\tau_{V}<1 \mathrm{~s}$, and the decays of $V$ inject electrons, muons, and mesons in numbers larger than baryons.

Dark photon decays with $m_{V} \leq 2 m_{\pi^{ \pm}}=279 \mathrm{MeV}$ result exclusively in injection of electromagnetic energy, because $V \rightarrow e^{+} e^{-}, \mu^{+} \mu^{-}$are the only kinematically accessible modes. Muons typically decay before interacting, and electron-positron pairs are quickly thermalized by interactions with background photons. The resulting electromagnetic cascade with spectrum $f_{\gamma}\left(E_{\gamma}\right)$ entails a large number of non-thermal photons that may then spall light elements.

Importantly, the spectrum has a relatively sharp cut-off for energies above the $e^{ \pm}$pair-creation threshold, $E_{\text {pair }} \simeq$ $m_{e}^{2} /(22 T)$. Photons with $E_{\gamma}>E_{\text {pair }}$ are being dissipated before they interact with nuclei, and to good approximation $f_{\gamma}\left(E_{\gamma}\right)=0$ for $E_{\gamma}>E_{\text {pair }}$. Photons with $E_{\gamma}<E_{\text {pair }}$, however, undergo slower degradation processes and may interact with the light elements before being thermalized. Equating $E_{\text {pair }}$ against the photo-destruction thresholds (in brackets below) yields the temperature and thereby the cosmic time $t_{\mathrm{ph}}$ of biggest impact for a spallation channel:

$t_{\mathrm{ph}} \simeq\left\{\begin{array}{llr}2 \times 10^{4} \mathrm{~s}, & { }^{7} \mathrm{Be}+\gamma \rightarrow{ }^{3} \mathrm{He}+{ }^{4} \mathrm{He} & (1.59 \mathrm{MeV}), \\ 5 \times 10^{4} \mathrm{~s}, & \mathrm{D}+\gamma \rightarrow n+p & (2.22 \mathrm{MeV}), \\ 4 \times 10^{6} \mathrm{~s}, & { }^{4} \mathrm{He}+\gamma \rightarrow{ }^{3} \mathrm{He} / \mathrm{T}+n / p & (20 \mathrm{MeV}),\end{array}\right.$

The spallation rate of species $N$ with number density $n_{N}$ is given by

$$
\Gamma_{\mathrm{ph}}(T)=2 n_{N} \int_{E_{\mathrm{thr}}}^{E_{\max }} d E_{\gamma} f_{\gamma}\left(E_{\gamma}\right) \sigma_{\gamma+N \rightarrow X}\left(E_{\gamma}\right),
$$

where $\sigma_{\gamma+N \rightarrow X}\left(E_{\gamma}\right)$ is the photo-dissociation cross section for $\gamma+N \rightarrow X$ with threshold $E_{\mathrm{thr}}$. The factor of two accounts for the two independent cascades that form in a back-to-back decay of $V$ at rest, each with a maximum energy of $E_{\max }=\max \left\{E_{\mathrm{pair}}, E_{\mathrm{inj}} / 2\right\}$. All spallation reactions listed in [14] are taken into account in the numerical analysis. We note in passing that neutrino injection

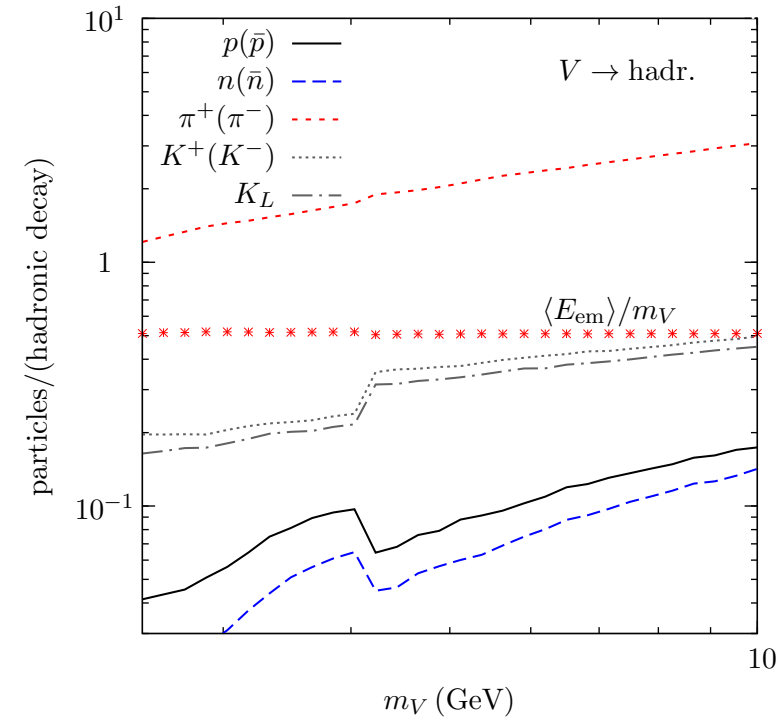

Figure 2. Pythia simulation on the average number of particles per $V$ decay and on the injected electromagnetic energy after all particles have decayed or annihilated to electrons and photons. About one third of the energy is carried away by neutrinos. Narrow hadronic resonances are neglected.

from muon decay constitute only minor corrections to the photon-induced processes listed above [5].

For vector masses above the di-pion threshold, $m_{V}>$ $2 m_{\pi^{ \pm}}$, hadronic modes are accessible in the decay of $V$ and the effects on BBN are more intricate. In the hadronic decay of $V$ only $\pi^{ \pm}, K^{ \pm}$, and $K_{L}$, with lifetimes $\tau \sim 10^{-8} \mathrm{~s}$, and (anti-)nucleons have a chance to undergo a strong interaction reaction before decaying by themselves.

Before deuterium formation at $T \simeq 100 \mathrm{keV}$, only charge exchange reactions on nucleons, such as $\pi^{-}+p \rightarrow$ $\pi^{0}+n$, are possible. They change the $n / p$ ratio and thereby most prominently the primordial ${ }^{4} \mathrm{He}$ value. After the deuterium bottleneck-once light elements have formedcharge exchange creates "extra neutrons" on top of the residual and declining neutron abundance. In addition, absorption with subsequent destruction of light elements such as $\pi^{-}+{ }^{4} \mathrm{He} \rightarrow T+n$ is now operative. Spallation of ${ }^{4} \mathrm{He}$ may also have a secondary consequence: the production of mass- 3 elements with non-thermal kinetic energy may induce reactions of the sort $\mathrm{T}+{ }^{4} \mathrm{He}_{\mathrm{bg}} \rightarrow{ }^{6} \mathrm{Li}+n$. In the numerical analysis, these processes as well as secondary populations of $\pi^{ \pm}$from kaon decays, and hyperon producing channels from reactions of kaons on nucleons and nuclei are being accounted for. Furthermore, in our analysis, we restrict ourselves to reactions at threshold, with charged pions and kaons being thermalized before reacting on light elements; such approximation generally results in more conservative constraints. A detailed quantitative discussion of incomplete stopping can be found in [5].

Finally, baryon pairs are produced in the $V$-decay for $m_{V} \gtrsim 2 \mathrm{GeV}$. Final state nucleons $\bar{n}$ and $\bar{p}$ will preferentially annihilate on protons with an annihilation cross section $\left\langle\sigma_{\mathrm{ann}} v\right\rangle \sim m_{\pi^{ \pm}}^{-2}$. The injection of $n \bar{n}$ then results in 


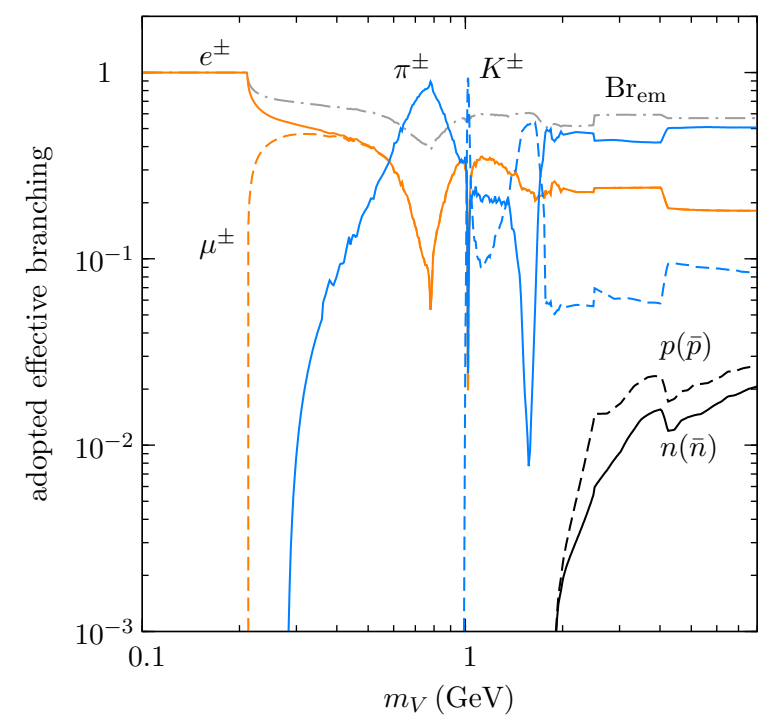

Figure 3. Final state branching ratios of long-lived mesons and other relevant decay products. BaBar measurements of the $e^{ \pm} \rightarrow$ $\pi^{ \pm}$and $e^{ \pm} \rightarrow K^{ \pm}$cross sections up to $m_{V}=1.8 \mathrm{GeV}$ are stiched together with a Pythia simulation starting at $m_{V} \geq 2.5 \mathrm{GeV}$; a branching to $K_{L}$ was neglected and the fraction of $m_{V}$ that is ultimately being converted to electromagnetic energy is labeled $\mathrm{Br}_{\mathrm{em}}$.

one net $p \rightarrow n$ conversion with associated energy injection of $m_{p}+m_{n}$. Annihilation on neutrons with similar cross section is also possible and $p \bar{p}$ injection results in one net $n \rightarrow p$ conversion. Assuming equal cross sections, the relative efficiency is $p /(n+p)$ and $n /(n+p)$, respectively.

At threshold, the rate for neutron injection can be inferred from a measurement of the $e^{+} e^{-} \rightarrow n \bar{n}$ cross section, $\sigma_{e^{+} e^{-} \rightarrow n \bar{n}} \sim 1 \mathrm{nb}$ [15]. Given a total hadronic cross section $\sigma_{e^{+} e^{-} \rightarrow \text { had }} \sim 50 \mathrm{nb}$ at this energy, the branching fraction to a neutron-antineutron pair is $\sim 2 \%$. Away from the di-nucleon threshold, with multi-pion(kaon) production and decays to hyperons and baryonic resonances being prevalent, $V$-decays may be simulated using Pythia. The ultimate yield of $\pi^{ \pm}, K^{ \pm}, K_{L}$, and nucleons prior to their decay is shown in Fig. 2; dots depict the average electromagnetic energy that is injected after all particles have decayed to electrons and photons; $e^{+}$have been annihilated on $e^{-}$. The rest of the decay-energy is carried away by neutrinos. At lower energies, decay events are eventually dominated by two body decays. Above the dipion (di-kaon) threshold, we use BaBar measurements of the $e^{ \pm} \rightarrow \pi^{ \pm}$and $e^{ \pm} \rightarrow K^{ \pm}$cross section until an reported energy of $\sqrt{s}=m_{V}=1.8 \mathrm{GeV}$. Relevant ultimate branching ratios are shown in Fig. 3; the effects of $K_{L}$ are, for simplicity, neglected in our BBN anaslysis.

A more detailed discussion along with a list of all included reactions can be found in the original paper [7] as well as in the preceding work [5]. Numerical results were obtained by usage of a Boltzmann code that is based on Ref. [16], with significant improvements and updates as detailed in [5]. Standard BBN yields are found to be in agreement with [17] when using a baryon asymmetry of $\eta_{b}=6.2 \times 10^{-10}$ and a neutron lifetime of $\tau_{n}=885.7 \mathrm{~s}$.

\subsection{Light element observations}

BBN sensitivity is attained by the observational inferrence of light element abundances and their estimated error bar. Here we briefly discuss those observations that form the basis of our obtained regions of interest.

The most abundant element after hydrogen is helium. Its mass fraction $Y_{p}$ is inferred from extragalactic HII regions, and values in the range

$$
0.24 \leq Y_{p} \leq 0.26
$$

have been reported over the years. Owing to potential systematic uncertainties $[18,19]$ we adopt (11) as the cosmologically viable range.

Among recent developments, the precision determination of $\mathrm{D} / \mathrm{H}$ from high redshift quasar absorption systems stands out [20, 21]. Error bars have reduced by a factor of five in comparison to previously available determinations. The weighted mean now reads [21],

$$
\mathrm{D} / \mathrm{H}=(2.53 \pm 0.04) \times 10^{-5} \text {. }
$$

$\mathrm{D}$ astration on dust grains is, however, a potential source of systematic uncertainty, and values as high as $4 \times 10^{-5}$ have also been reported [22, 23]. In light of this, we adopt an upper limit of,

$$
\mathrm{D} / \mathrm{H}<3 \times 10^{-5}
$$

as well. Finally, producing too little $\mathrm{D} / \mathrm{H}$ yields a robust limit because no known astrophysical sources of this fragile light element exist. We therefore either use the nominal lower $2 \sigma$-limit from (12) or require (robustly),

$$
{ }^{3} \mathrm{He} / \mathrm{D}<1
$$

instead. The latter value is derived form solar system observations [24].

Finally, and with much smaller abundance, the primordial value of ${ }^{7} \mathrm{Li} / \mathrm{H}$ [25], is lower than the lithium yield from standard $\mathrm{BBN}$ by a factor of $3-5,{ }^{7} \mathrm{Li} / \mathrm{H}=$ $\left(5.24_{-0.67}^{+0.71}\right) \times 10^{-10}$ [17]. We consider lithium being in concordance with observations if BBN predictions yield

$$
10^{-10}<{ }^{7} \mathrm{Li} / \mathrm{H}<2.5 \times 10^{-10} .
$$

While new physics may be at the heart of the lithium problem, we caution that astrophysical depletion mechanisms may also play their part in solution to this long-standing puzzle; see [26] for a recent review.

\subsection{Results}

Our results from $V$-decays and their effect on BBN are presented in the $m_{V}, \kappa$ parameter space in Fig. 4. Contours of constant lifetime, $\tau_{V}$ and freeze-in abundance $n_{V} / n_{b}$ are shown by the diagonal solid and dotted lines, respectively. 


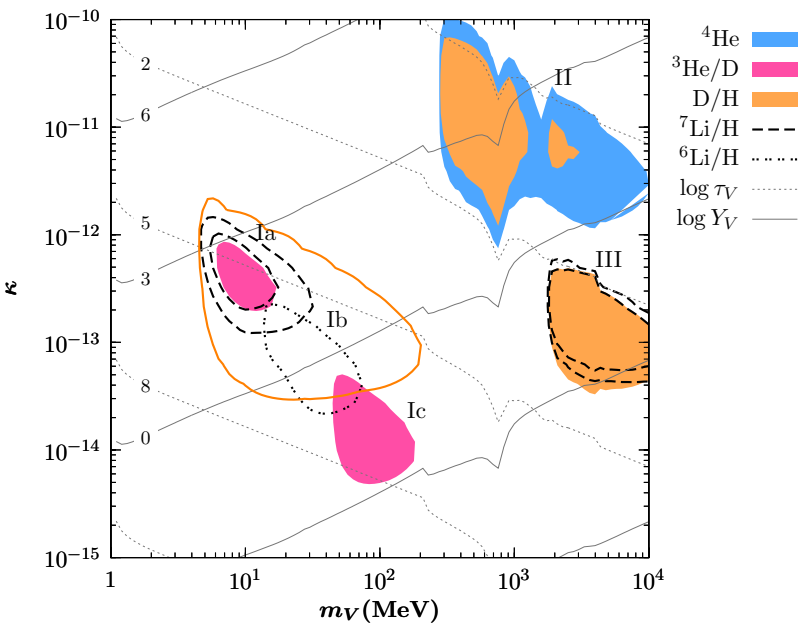

Figure 4. Vector mass $m_{V}$ and kinetic mixing parameter $\kappa$ parameter space with BBN sensitivity. Diagonal gray contours depict $\tau_{V}$ (solid) or $n_{V} / n_{b}$ prior to decay (dotted). Shaded regions are excluded by observations as labeled. The solid (orange) closed line is a $2 \sigma$ constraint from underproduction of $\mathrm{D} / \mathrm{H}$ derived from (12). Dashed black lines show decreasing levels of ${ }^{7} \mathrm{Li} / \mathrm{H}, 4 \times 10^{-10}$ and $3 \times 10^{-10}$, from outer cirlces to the inner ones, respectively. Along the dotted line ${ }^{6} \mathrm{Li} / \mathrm{H}=10^{-12}$ signifying an two orders magnitude enhanced ${ }^{6} \mathrm{Li}$ yield, that is, however, not yet constrained by observations.

The regions labeled I-III are in conflict with observations as detailed in the previous section.

In regions I, $V$ decays to $e^{+} e^{-}$result in electromagnetic energy injection. Region Ia $\left(\tau_{V} \sim 10^{5} \mathrm{~s}\right)$ is marked by a destruction of ${ }^{7} \mathrm{Be}$ and $\mathrm{D}$. The ${ }^{7} \mathrm{Li} / \mathrm{H}$ abundance is reduced to $4 \times 10^{-10}$ and $3 \times 10^{-10}$ from the outside to the inside, respectively. However, cosmologically favored smaller ${ }^{7} \mathrm{Li} / \mathrm{H}$ abundances are challenged by ${ }^{3} \mathrm{He} / \mathrm{D}<1$ (pink shaded region). Using (12), lower ${ }^{7} \mathrm{Li} / \mathrm{H}$ values are excluded by the nominal $2 \sigma$ lower limit on $\mathrm{D} / \mathrm{H}$ as depicted by the solid closed line. Region Ib is additionally marked by spallation of ${ }^{7} \mathrm{Li}$ and ${ }^{7} \mathrm{Be}$ from non-thermal photons. This results in direct production of ${ }^{6} \mathrm{Li} / \mathrm{H}>10^{-12}$ —values yet too low for being observationally constrained at the moment. Finally, Region Ic with $\tau_{V} \sim 10^{7} \mathrm{~s}$ is marked by ${ }^{4} \mathrm{He}$ dissociation and net creation of ${ }^{3} \mathrm{He} / \mathrm{D}$ ruling out this parameter region. Secondary production of ${ }^{6} \mathrm{Li}$ is not efficient enough to yield an additional limit.

In region II, $\tau_{V}<100 \mathrm{~s}$ and $V$ decays before the end of the D-bottleneck $(T \sim 100 \mathrm{keV})$. Injection of pions, kaons, and nucleons, results in anomalous $n \leftrightarrow p$ inter-conversion. The consequence is an elevated $n / p$ ratio and therefore enhanced $\mathrm{D}$ and ${ }^{4} \mathrm{He}$ yields. The lowlifetime/high-abundance region II is correspondingly disfavored by $Y_{p} \leq 0.26$ and $\mathrm{D} / \mathrm{H} \leq 3 \times 10^{-5}$.

Finally, region III is marked by the production of "extra neutrons" at $t \sim 10^{3} \mathrm{~s}$ from $V \rightarrow n \bar{n}$ and from charge exchange of $\pi^{-}$on protons, $\pi^{-} p \rightarrow n \pi^{0}$ or $\pi^{-} p \rightarrow n \gamma$. In addition, hyperon production by "s-quark" exchange of $K^{-}$on protons may also result in extra neutrons. With it comes a path that may deplete lithium, ${ }^{7} \mathrm{Be}+n \rightarrow{ }^{7} \mathrm{Li}+p$, followed by ${ }^{7} \mathrm{Li}+p \rightarrow{ }^{4} \mathrm{He}+{ }^{4} \mathrm{He}$. With a reduced Coulomb barrier, ${ }^{7} \mathrm{Li}$ is more susceptible to proton burning in the second step and the declining ${ }^{7} \mathrm{Li}$ trend is depicted by the dashed lines in Fig. 4. Most of the extra neutrons, however, end up being captured by protons and the associated $\mathrm{D} / \mathrm{H}$ constraint (13) is given by the orange region.

\section{Conclusions}

The kinetic mixing of a new $U(1)^{\prime}$ gauge group with the Standard Model $U(1)$ factors of hypercharge and, below the electroweak scale, of electromagnetism is one of the few portals to the hidden sector with renormalizable couplings. The associated gauge boson $V$ is often called a "dark photon" and in this manuscript we have reported the cosmological limits from BBN as they have been derived in [7]. BBN sensitivity reaches photon kinetic mixing parameters of $\kappa \sim 10^{-14}$ for $1 \mathrm{MeV} \leq m_{V} \lesssim 10 \mathrm{GeV}$, unchallenged from terrestrial dark photon searches, see, e.g. the works and presentations [27-33]

The presented limits are based on a thermal abundance of $V$ and a standard cosmological history of the Universe-i.e. uneventful until $V$-decay-with reheat temperatures in excess of $m_{V}$. Additional contributions to the $V$-abundance such as from an initial $V$-condensate after inflation may only strengthen the derived bounds. The latter source of primordial $V$-particles is particularly interesting in the context of smaller $V$-masses. Below the di-electron threshold (not considered in this work), $V$ has a naturally long lifetime with $V \rightarrow 3 \gamma$ being the only decay mode. Therefore $V$ can even be a dark matter candidate $[6,34]$ and ensuing constraints on the photoelectric absorption of $V$ on atoms in dark matter detectors have started to receive attention only very recently $[35,36]$

\section{Acknowledgements}

The speaker thanks S. Eidelman for the invitation to the workshop. JP is supported by the New Frontiers program by the Austrian Academy of Sciences.

\section{References}

[1] M. Pospelov and J. Pradler, Ann. Rev. Nucl. Part. Sci. 60, 539 (2010) [arXiv:1011.1054 [hep-ph]].

[2] R. Essig, J. A. Jaros, W. Wester, P. H. Adrian, S. Andreas, T. Averett, O. Baker and B. Batell et al., arXiv:1311.0029 [hep-ph].

[3] B. Holdom, Phys. Lett. B 166, 196 (1986).

[4] B. Batell, M. Pospelov and A. Ritz, Phys. Rev. D 79, 115008 (2009) [arXiv:0903.0363 [hep-ph]].

[5] M. Pospelov and J. Pradler, Phys. Rev. D 82, 103514 (2010) [arXiv:1006.4172 [hep-ph]].

[6] J. Redondo and M. Postma, JCAP 0902, 005 (2009) [arXiv:0811.0326 [hep-ph]].

[7] A. Fradette, M. Pospelov, J. Pradler and A. Ritz, Phys. Rev. D 90, 035022 (2014) [arXiv:1407.0993 [hep-ph]].

[8] H. An, M. Pospelov and J. Pradler, Phys. Lett. B 725, 190 (2013) [arXiv:1302.3884 [hep-ph]]. 
[9] H. An, M. Pospelov and J. Pradler, Phys. Rev. Lett. 111, 041302 (2013) [arXiv:1304.3461 [hep-ph]].

[10] J. Redondo and G. Raffelt, JCAP 1308, 034 (2013) [arXiv:1305.2920 [hep-ph]].

[11] J. P. Lees et al. [BaBar Collaboration], Phys. Rev. D 86, 032013 (2012) [arXiv:1205.2228 [hep-ex]].

[12] J. P. Lees et al. [BaBar Collaboration], Phys. Rev. D 88, no. 3, 032013 (2013) [arXiv:1306.3600 [hep-ex]].

[13] E. Braaten and D. Segel, Phys. Rev. D 48, 1478 (1993) [hep-ph/9302213].

[14] R. H. Cyburt, J. R. Ellis, B. D. Fields and K. A. Olive, Phys. Rev. D 67, 103521 (2003) [astro-ph/0211258].

[15] A. Antonelli, R. Baldini, P. Benasi, M. Bertani, M. E. Biagini, V. Bidoli, C. Bini and T. Bressani et al., Nucl. Phys. B 517, 3 (1998).

[16] L. Kawano, FERMILAB-PUB-92-004-A.

[17] R. H. Cyburt, B. D. Fields and K. A. Olive, JCAP 0811, 012 (2008) [arXiv:0808.2818 [astro-ph]].

[18] Y. I. Izotov and T. X. Thuan, Astrophys. J. 710, L67 (2010) [arXiv:1001.4440 [astro-ph.CO]].

[19] E. Aver, K. A. Olive, R. L. Porter and E. D. Skillman, JCAP 1311, 017 (2013) [arXiv:1309.0047 [astroph.CO]].

[20] M. Pettini and R. Cooke, Mon. Not. Roy. Astron. Soc. 425, 2477 (2012) [arXiv:1205.3785 [astroph.CO]].

[21] R. Cooke, M. Pettini, R. A. Jorgenson, M. T. Murphy and C. C. Steidel, arXiv:1308.3240 [astro-ph.CO].

[22] S. Burles and D. Tytler, Astrophys. J. 507, 732 (1998) [astro-ph/9712109].

[23] D. Kirkman, D. Tytler, N. Suzuki, J. M. O’Meara and D. Lubin, Astrophys. J. Suppl. 149, 1 (2003) [astro- ph/0302006].

[24] Prantzos, N., Vangioni-Flam, E. and Cassé, M. 1993, Origin and evolution of the elements. Proceedings., Cambridge University Press, Cambridge (UK), 1993

[25] F. Spite and M. Spite, Astron. Astrophys. 115, 357 (1982).

[26] B. D. Fields, Ann. Rev. Nucl. Part. Sci. 61, 47 (2011) [arXiv:1203.3551 [astro-ph.CO]].

[27] J. Beacham, EPJ Web Conf. 73, 07011 (2014).

[28] A. Celentano [the HPS Collaboration], J. Phys. Conf. Ser. 556, no. 1, 012064 (2014).

[29] M. Battaglieri et al. [BDX Collaboration], arXiv:1406.3028 [physics.ins-det].

[30] F. Curciarello [KLOE/KLOE-2 Collaboration], EPJ Web Conf. 72, 00004 (2014).

[31] H. Merkel [A1 Collaboration], EPJ Web Conf. 81, 01020 (2014).

[32] M. Raggi and V. Kozhuharov, Adv. High Energy Phys. 2014, 959802 (2014) [arXiv:1403.3041 [physics.ins-det]].

[33] R. Essig, P. Schuster, N. Toro and B. Wojtsekhowski, JHEP 1102, 009 (2011) [arXiv:1001.2557 [hep-ph]].

[34] M. Pospelov, A. Ritz and M. B. Voloshin, Phys. Rev. D 78, 115012 (2008) [arXiv:0807.3279 [hep-ph]].

[35] K. Abe et al. [XMASS Collaboration], Phys. Rev. Lett. 113, 121301 (2014) [arXiv:1406.0502 [astroph.CO]].

[36] H. An, M. Pospelov, J. Pradler and A. Ritz, arXiv:1412.8378 [hep-ph]. 